Mathematical Research Letters 3, 1-17 (1996)

\title{
ZETA FUNCTIONAL DETERMINANTS ON MANIFOLDS WITH BOUNDARY
}

\author{
Sun-Yung A. Chang and Jie QIng
}

\begin{abstract}
A в sтRAст. This article is an announcement of our recent work on the zeta functional determinants on manifolds with boundary. We first derive some geometric formulas for the quotient of the zeta functional determinants for certain elliptic boundary value problems on Riemannian 3 \& 4-manifolds with smooth boundary. We then apply the formulas to establish $W^{2,2}$ compactness of isospectral set within a subclass of conformal metrics, and to prove some existence and uniqueness properties of extremal metrics for the zeta functional determinants. Some key elements in our proof include the discovery of some boundary operator conformal covariant of degree 3 and establishment of some sharp Sobolev trace inequalities of LebedevMilin type.
\end{abstract}

\section{Part 1. Alvarez-Polyakov formula}

\subsection{Preliminaries}

Let $M$ be a compact Riemannian manifold with smooth boundary $\partial M$. We shall consider boundary value problems $(A, B)$ satisfying the three basic assumptions below. For simplicity we shall only consider operators acting on the space of functions on $M$, although all the statements in this article could be formulated for operators acting on the space of sections of a tensorspinor bundle over $M$.

Analytic Assumptions. Let $A$ be a differential operator of order 2l. Suppose that $A$ is formally self-adjoint and has positive definite leading symbol. Let $B$ be an operator for the Cauchy data for $A$ on $\partial M$ such that the pair $(A, B)$ is elliptic.

Received November 8, 1995.

Research of the first author is supported in part by NSF Grant DMS-9401465.

Research of the second author is supported in part by NSF Grant DMS-9407646. 
Naturality Assumptions. Suppose that $A$ and $B$ are geometric differential operators on $(M, \partial M)$ : i.e. they are defined independently of the choices of local coordinates on $(M, \partial M)$.

If $(A, B)$ satisfies the above two assumptions, then for any $f \in C^{\infty}(\bar{M})$,

$$
\operatorname{Tr}_{L^{2}} f \exp \left(-t A_{B}\right) \sim \sum_{n=0}^{\infty} a_{n}(f, A, B) t^{\frac{n-m}{2 l}}, t \rightarrow 0^{+},
$$

where

$$
a_{n}(f, A, B)=\int_{M} f I_{n}(A) d x+\sum_{i=0}^{n-1} \oint_{\partial M} N^{i} f B_{n, i}(A, B) d y
$$

for some interior local invariant $I_{n}(A)$ of order $n$ and some boundary local invariants $B_{n, i}(A, B)$ of order $n-i-1$, where $N^{i} f$ is the $i$ th covariant derivative of $f$ in the inward normal direction on the boundary $\partial M$. Let

$$
\zeta_{(A, B)}(s)=\sum_{\lambda_{j} \neq 0}\left|\lambda_{j}\right|^{-s}
$$

where $\lambda_{j}$ are all eigenvalues of the boundary value problem $(A, B)$. Clearly this zeta function is only well-defined as above for $s$ with sufficient large real part. But it can be regularized by meromorphic continuation (cf., [RS], $[\mathrm{BG}-1])$. In particular, $\zeta_{(A, B)}(s)$ is regular at $s=0$, then one formly defines

$$
\log \operatorname{det}(A, B)=-\zeta_{(A, B)}^{\prime}(0)
$$

In general, the zeta functional determiant defined as above is an absolute global quantity. But its variation, on the other hand, is a local geometric quantity. We therefore consider families of conformal metrics on $(M, \partial M)$ and require $(A, B)$ satisfy a third assumption as in the following. We use the notation that a geometric quantity $\mathcal{Q}$ at the metric $g_{\omega}=e^{2 \omega} g_{0}$ is denoted by $\mathcal{Q}[\omega]$.

Conformal Assumptions. Suppose that both $A$ and $B$ are conformally covariant of bidegree $\left(a_{1}, a_{2}\right)$ and $\left(b_{1}, b_{2}\right)$ in the following sense

$$
\begin{aligned}
& A[\omega](f)=e^{-a_{1} \omega} A[0]\left(e^{a_{2} \omega} f\right) \\
& B[\omega](g)=e^{-b_{1} \omega} B[0]\left(e^{b_{2} \omega} g\right),
\end{aligned}
$$

for any $f \in C^{\infty}(M), g \in C^{\infty}(\partial M)$. And assume that

$$
B[0]\left(e^{a_{2} \omega} g\right)=0 \text { if and only if } B[\omega](g)=0,
$$


for any $\omega \in C^{\infty}(\bar{M})$.

Example. The very typical examples of pairs $(A, B)$ which satisfy all three assumptions above are

$$
A=\mathcal{L}=\Delta+\frac{m-2}{4(m-1)} \tau
$$

the conformal Laplacian of bidegree $\left(\frac{m+2}{2}, \frac{m-2}{2}\right)$, where $\tau$ is the scalar curvature, and $B$ is either the Dirichlet boundary condition or

$$
B=\mathcal{R}=-N+\frac{m-2}{2(m-1)} H
$$

the Robin operator of bidegree $\left(\frac{m}{2}, \frac{m-2}{2}\right)$, where $H$ is the mean curvature on $\partial M$.

Theorem 1.1.1(Branson and Gilkey [BG-1]). Suppose that $(A, B)$ satisfies above analytic, naturality, and conformal assumptions. Then

$$
\frac{d}{d \epsilon} a_{m}(1, A, B)[\omega+\epsilon \eta]_{\left.\right|_{\epsilon=0}}=0
$$

and

$$
\frac{d}{d \epsilon} \zeta_{(A, B)[\omega+\epsilon \eta]}^{\prime}(0)_{\left.\right|_{\epsilon=0}}=2 l\left\{a_{m}(\eta, A, B)[\omega]-\sum_{\lambda_{j}[\omega]=0} \int_{M} \eta\left|\phi_{j}[\omega]\right|^{2} d x[\omega]\right\}
$$

where $\phi_{j}[\omega]$ are normalized eigenfunctions of $(A, B)[\omega]$ with eigenvalues $\lambda_{j}[\omega]$.

In cases when $(A, B)$ have no zero eigenvalues, if we denote $\log \frac{\operatorname{det}(A, B)[\omega]}{\operatorname{det}(A, B)[0]}$ by $F[\omega]$,

$$
F_{t}^{\prime}[t \omega]=-2 l a_{m}(\omega, A, B)[t \omega]
$$

by (1.1.6), where the right hand side is a polynomial in $t$ of order at most $m$. Thus integrating with respect to $t$ from 0 to 1 , one finds the formula of $F[\omega]$. Recently Branson and Gilkey in [BG-1] [BG-3] have used this method to compute $F[\omega]$ for differential operators $(\mathrm{A}, \mathrm{B})$ defined on compact manifolds of dimension 4. But the expression of $F[\omega]$ computed using this method will not in general be in geometric form. 


\subsection{Alvarez-Polyakov formulas in Dimension 2 \& 3}

In dimension two, Alvarez [Al] considered the Laplacian with the Dirichlet boundary condition on compact surfaces with boundary and derived the following Polyakov (cf. [Po] [RS]) type formula:

$$
\begin{aligned}
\log \frac{\operatorname{det} \Delta[\omega]}{\operatorname{det} \Delta[0]}= & -\frac{1}{6 \pi}\left\{\frac{1}{2} \int_{M} \omega \Delta \omega+\int_{M} \omega K[0]+\frac{1}{2} \oint_{\partial M} \omega(-N \omega)\right. \\
& \left.+\oint_{\partial M} \omega k[0]\right\}-\frac{1}{4 \pi}\left\{\oint_{\partial M}(k d s)[\omega]-\oint_{\partial M}(k d s)[0]\right\},
\end{aligned}
$$

where $K$ is the Gaussian curvature of $M$ and $k$ is the geodesic curvature of $\partial M$. Hence we are looking for Alvarez-Polyakov formula in higher dimensions.

To compute Alvarez-Polyakov formula for boundary value problem $(A, B)$ in dimension three, we choose bases of local scalar invariants and express the heat coefficient $a_{3}$ as follows:

$$
\begin{aligned}
a_{3}(f, A, B)= & \oint_{\partial M} f\left\{\alpha_{1} \tilde{K}+\alpha_{2} H^{2}+\alpha_{3}|L|^{2}+\alpha_{4} F\right\} d y \\
& +\oint_{\partial M}\left\{\beta f_{; N} H+\gamma f_{; N N}\right\} d y
\end{aligned}
$$

where $F=R_{a N a N}$ is a partial trace of Riemannian curvature, $\tilde{K}$ is the Gaussian curvature on $\partial M$ and $L$ is the second fundamental form of $\partial M$. Then using transformation formulas for all local scalar invariants and local argumented scalar invariants under conformal changes of metrics and (1.1.5) we may conclude

$$
\begin{aligned}
a_{3}(\eta, A, B)= & \oint_{\partial M} \eta\left\{\alpha_{1} \tilde{K}+\alpha_{2}\left(H^{2}-2|L|^{2}\right)\right\} d s \\
& +\oint_{\partial M}\left\{\beta \eta_{; N} H+\gamma \eta_{; N N}\right\} d s .
\end{aligned}
$$

In particular, there are only four undetermined coefficients in the above expression of $a_{3}$. Furthermore we can express $a_{3}$ as a conformal variation and as a consequence, we can derive the following result.

Theorem 1.2.1. Let $M$ be a compact 3-manifold with smooth boundary. Under the three assumptions and that $(A, B)$ has no zero eigenvalue, one 
has

$$
\begin{aligned}
& -\frac{1}{2 l} \log \frac{\operatorname{det}(A, B)[\omega]}{\operatorname{det})(A, B)[0]}= \\
& \alpha_{1}\left\{\frac{1}{2} \oint_{\partial M}|\tilde{\nabla} \omega|^{2} d y+\oint_{\partial M} \tilde{K}[0] \omega d y\right\}+\alpha_{2} \oint_{\partial M} \omega\left(\left(H^{2}-2|L|^{2}\right) d y\right)[0] \\
& +\frac{1}{4} \beta\left\{\oint_{\partial M}\left(H^{2} d s\right)[0]-\oint_{\partial M}\left(H^{2} d s\right)[\omega]\right\}+\frac{1}{2} \gamma\left\{\oint_{\partial M}\left(\left(F+\frac{1}{4} H^{2}\right) d s\right)[0]\right. \\
& \left.-\oint_{\partial M}\left(\left(F+\frac{1}{4} H^{2}\right) d s\right)[\omega]\right\} .
\end{aligned}
$$

We would like to remark that some preliminary version of the formula (1.2.4) has appeared in [B-3].

\subsection{Alvarez-Polyakov formula in Dimension 4}

In dimension 4 , it is known that a curvature quantity $Q=-2|V|^{2}+$ $2 J^{2}+\Delta J$, (where $V=(\tau-J g) / 2$ and $\left.J=\tau / 6\right)$, called Paneitz quantity (cf. $[\mathrm{BO}][\mathrm{BCY}][\mathrm{CY}]$ ), plays a similar role as the Gaussian curvature does in dimension 2. The differential operator associated with the Paneitz quantity is $P_{4}=\Delta^{2}+\delta\{2 J-4 V \cdot\} d$, called Paneitz operator, which appears in the transformation formula of $Q$ under conformal change of metrics as follows:

$$
P_{4} \omega+Q[0]=Q[\omega] e^{4 \omega},
$$

like the one in which the Gaussian curvature $K$ and the Laplacian $P_{2}=\Delta$ are involved in dimension 2. Another curvature which has appeared in the Alvarez-Polyakov formula in dimension 2 is the geodesic curvature $k$ of the boundary, which is associated with the Neumann operator $P_{1}=-N$ in the following way:

$$
-N \omega+k[0]=k[\omega] e^{\omega} \text { on } \partial M
$$

In dimension 4, we have discovered a boundary curvature invariant

$$
T=-\frac{1}{2} N J+J H-R_{a N b N} L_{a b}+\frac{1}{9} H^{3}-\frac{1}{3} \operatorname{Tr} L^{3}+\frac{1}{3} \tilde{\Delta} H,
$$

and its associated operator

$$
P_{3}=-\frac{1}{2} N \Delta-\tilde{\Delta} N+\frac{2}{3} H \tilde{\Delta}+L_{a b} \tilde{\nabla}_{a} \tilde{\nabla}_{b}+\frac{1}{3} \tilde{\nabla} H \cdot \tilde{\nabla}+(F-2 J) N .
$$


The curvature $T$ transforms under conformal change of metrics as

$$
P_{3} \omega+T[0]=T[\omega] e^{3 \omega},
$$

like (1.3.1) and (1.3.2). Chern-Gauss-Bonnet formula for compact 4- manifolds with boundary may be written as

$$
\chi(M)=\left(32 \pi^{2}\right)^{-1} \int_{M}\left(|C|^{2}+4 Q\right) d x+\left(4 \pi^{2}\right)^{-1} \oint_{\partial M}\left(T-\mathcal{L}_{4}-\mathcal{L}_{5}\right) d y,
$$

where $\mathcal{L}_{4}, \mathcal{L}_{5}$ are some pointwise conformally invariant boundary curvatures. Actually, the original term which has appeared in the Chern-GaussBonnet formula was the term $\mathcal{S}$ in the place of $T$, where $T=\mathcal{S}+\frac{1}{3} \tilde{\Delta} H$. In the second part of this article we shall see that the discovery of the pair $\left(P_{3}, T\right)$ is very crucial for us to establish some sharp Sobolev Trace inequality of Lebedev-Milin type.

Following the definition of $P_{3}$ and $T$, we may re-write the formula of heat coefficient $a_{4}$ given by Branson and Gilkey (cf. [BG-1]) into the following form.

$$
\begin{aligned}
& a_{4}(\eta, A[\omega], B[\omega])= \\
& \beta_{1} \int_{M} \eta\left(|C|^{2} d v\right)[\omega]+\beta_{2}\left\{\frac{1}{2} \int_{M} \eta(Q d v)[\omega]+\oint_{\partial M} \eta(T d s)[\omega]\right\} \\
& +\beta_{4} \oint_{\partial M} \eta\left(\mathcal{L}_{4} d s\right)[\omega]+\beta_{3}\left\{\int_{M} \eta(\Delta J)[\omega]-\oint_{\partial M} \eta\left(J_{N} d s\right)[\omega]\right\} \\
& \left.+\oint_{\partial M}\left(J \eta_{N} d s\right)[\omega]\right\}+\beta_{5} \oint_{\partial M} \eta\left(\mathcal{L}_{5} d s\right)[\omega]+\beta_{6} \oint_{\partial M} \eta(\tilde{\Delta} H d s)[\omega] \\
& +\beta_{7} \oint_{\partial M} \eta\left(R_{a N ; a} d s\right)[\omega]+\gamma_{1} \oint_{\partial M}\left(J \eta_{N} d s\right)[\omega]+\gamma_{2} \oint_{\partial M}\left(F \eta_{N} d s\right)[\omega] \\
& +\gamma_{3} \oint_{\partial M}\left(H^{2} \eta_{N} d s\right)[\omega]+\gamma_{4} \oint_{\partial M}\left(|L|^{2} \eta_{N} d s\right)[\omega]+\gamma_{5} \oint_{\partial M}\left(H \eta_{; N N} d s\right)[\omega] \\
& \left.+\gamma_{6} \oint_{\partial M} \eta_{; N N N} d s\right)[\omega] .
\end{aligned}
$$

Notice that there are 13 undetermined coefficients in (1.3.4). Our goal is to write $a_{4}$ as conformal variations of some geometric functionals. To be able to do so, it turns out a key observation is the proposition below following the definition.

Definition 1.3.1. A functional $F[\omega]$ of a metric $g_{\omega}=e^{2 \omega} g_{0}$ on a given conformal class is a conformal primitive of another functional $G_{\eta}[\omega]$ if

$$
\frac{d}{d \epsilon} F[\omega+\epsilon \eta]_{\left.\right|_{\epsilon=0}}=G_{\eta}[\omega]
$$

for all $\eta, \omega \in C^{\infty}(M)$. 
Proposition 1.3.2. Let $M$ be a compact Riemannian manifold of dimension $m$ with smooth boundary. Suppose that $(A, B)$ satisfies analytic, naturality and conformal assumptions. Then the heat coefficient $a_{m}(\eta, A[\omega]$, $B[\omega])$ has a conformal primitive.

Clearly a functional $G_{\eta}[\omega]$ that possesses a conformal primitive is rather restricted. Theorem 1.1.1 of Branson and Gilkey implies that the zeta functional determinant is a conformal primitive of $a_{4}$ if there is no zero eigenvalue for $(A, B)$. Our proposition here simply says that $a_{4}$ possesses a conformal primitive regardless of existence of zero eigenvalues. As a consequence of the above proposition and some lengthy computations we obtain the following result.

Lemma 1.3.3. Let $M$ be a compact 4-manifold with smooth boundary. Suppose $(A, B)$ satisfy the three assumptions. Then the undetermined coefficients in the expression (1.3.4) of $a_{4}$ satisfy

$$
\begin{array}{r}
\beta_{2}+3 \beta_{6}-2 \beta_{7}-2 \gamma_{2}+3 \gamma_{5}+4 \gamma_{6}=0 \\
-3 \beta_{6}+2 \beta_{7}-2 \gamma_{1}-4 \gamma_{2}+3 \gamma_{5}=0
\end{array}
$$

In other words, there are only 11 undetermined coefficients in $a_{4}$.

Equations (1.3.5) not only allow us to reduce the number of undetermined coefficients in the expression of $a_{4}$, but also enable us to write $a_{4}$ as a variation of some rather geometric quantities. After choosing a preferred basis of boundary local scalar invariants we arrive at the following Alvarez-Polyakov type formula.

Theorem 1.3.4. Let $M$ be a 4-manifold with smooth boundary. Suppose that $(A, B)$ satisfies analytic, naturality and conformal assumptions and that $(A, B)$ has no zero eigenvalues. Then

$$
\begin{aligned}
& -\frac{1}{2 l} \log \frac{\operatorname{det}(A, B)[\omega]}{\operatorname{det}(A, B)[0]}=\beta_{1} \int_{M} \omega\left(|C|^{2} d v\right)[0]+\beta_{2} \int_{M} D[\omega] d x[0] \\
& +\frac{1}{2} \beta_{3}\left\{\int_{M}\left(J^{2} d v\right)[\omega]-\int_{M}\left(J^{2} d v\right)[0]\right\}+\beta_{4} \oint_{\partial M} \omega\left(\mathcal{L}_{4} d s\right)[0] \\
& +\beta_{5} \oint_{\partial M} \omega\left(\mathcal{L}_{5} d s\right)[0]+\mu_{1} \oint_{\partial M}((T d s)[\omega]-(T d s)[0])
\end{aligned}
$$




$$
\begin{aligned}
& +\mu_{2} \oint_{\partial M}((\tilde{\tau} H d s)[w]-(\tilde{\tau} H d s)[0])+\mu_{5} \oint_{\partial M}\left(\left(H^{3} d s\right)[\omega]-\left(H^{3} d s\right)[0]\right) \\
& +\mu_{3} \oint_{\partial M}(((\tau H-3 F H) d s)[\omega]-((\tau H-3 F H) d s)[0]) \\
& +\mu_{4} \oint_{\partial M}\left(\left(\left(F H-3 R_{a N b N} L_{a b}\right) d s\right)[\omega]-\left(\left(F H-3 R_{a N b N} L_{a b}\right) d s\right)[0]\right) \\
& +\mu_{6} \oint_{\partial M}\left(\left(\left(H^{3}-9 \operatorname{Tr} L^{3}\right) d s\right)[\omega]-\left(\left(H^{3}-9 \operatorname{Tr} L^{3}\right) d s\right)[0]\right)
\end{aligned}
$$

where

$$
\begin{aligned}
D[\omega] & =\frac{1}{4} \int_{M} \omega P_{4} \omega d v+\frac{1}{2} \int_{M} \omega Q d v \\
& +\frac{1}{2} \oint_{\partial M} \omega P_{3} \omega d s+\oint_{\partial M} \omega T d s \\
& +\frac{1}{12}\left\{\oint_{\partial M}\left(-\tau-\frac{1}{3} H^{2}+3 F\right) \omega_{N} d s-\oint_{\partial M} H \omega_{; N N} d s-\oint_{\partial M} H \tilde{\Delta} \omega d s\right\},
\end{aligned}
$$

for 11 constants $\left\{\beta_{\nu}\right\}_{\nu=1}^{5}$ and $\left\{\mu_{i}\right\}_{i=1}^{6}$ depending only on $(A, B)$.

Example. Suppose that $A=\mathcal{L}$ the conformal Laplacian and $B=\mathcal{R}$ the Robin operator. Then, we have up to a common scale (cf. [BG-2]):

\begin{tabular}{|l|c|c|c|}
\hline$\beta_{1}=2$ & $\beta_{2}=-8$ & $\beta_{3}=-8$ & $\beta_{4}=-4$ \\
\hline$\beta_{5}=-8$ & $\mu_{1}=60$ & $\mu_{2}=-\frac{4}{3}$ & $\mu_{3}=-\frac{50}{9}$ \\
\hline$\mu_{4}=-20$ & $\mu_{5}=-\frac{22}{9}$ & $\mu_{6}=-\frac{44}{27}$. & \\
\hline
\end{tabular}

Table 1.3.8

\section{Part 2. Compactness results and extremal metrics}

\subsection{Introduction}

Considering the pair $(\tau, H)$ in dimension larger than 2 , one finds that the total curvature of $(M, \partial M)$ is a conformal primitive of the pair $(\tau, H)$ in the following sense:

$$
\begin{aligned}
\left.\frac{d}{d \epsilon}\right|_{\epsilon=0}\left\{\int_{M}(\tau d v)[\omega+\epsilon \eta]+2 \oint_{\partial M}(H d s)[\omega+\epsilon \eta]\right\} \\
=(m-2)\left\{\int_{M} \eta(\tau d v)[\omega]+2 \oint_{\partial M} \eta(H d s)[\omega]\right\} .
\end{aligned}
$$


Let us name the conformal change of metrics preserving the $\operatorname{vol}(M)$ as the conformal variation of type $I$, and name the conformal change of metrics preserving the $\operatorname{vol}(\partial M)$ as the conformal variation of type II. Then an extremum of the total curvature under conformal variations of type I is achieved by metrics with constant scalar curvature inside and vanishing mean curvature on the boundary; and its extremum under conformal variations of type II is achieved by metrics with vanishing scalar curvature and constant mean curvature. Escobar in [E-1] [E-2] [E-3] has established certain sharp Sobolev trace inequalities on manifolds with boundary and obtained the existences of extrema of the total curvature under variations of both types. In particular, he has established (independently by Beckner [Be-1]) the following result.

$$
\int_{M}(\tau d v)[\omega]+2 \oint_{\partial M}(H d s)[\omega]-\int_{M}(\tau d v)[0]+2 \oint_{\partial M}(H d s)[0] \geq 0
$$

for either $(M, g)=\left(S_{+}^{m}, g_{0}\right)$ and $\operatorname{vol}(M)[\omega]=\operatorname{vol}(M)[0]$, or $(M, g)=$ $\left(B^{m}, g_{0}\right)$ and $\operatorname{vol}(\partial M)[\omega]=\operatorname{vol}(\partial M)[0]$, equality holds if and only if $g_{w}$ is isometric to $g_{0}$, the standard canonical metric on $S_{+}^{m}$ or $B^{m}$.

In dimension 2 , for the pair $(K, k)$, one finds that instead the zeta functional determinant of the Laplacian with Dirichlet boundary condition (see the Alvarez-Polyakov formula (1.2.1)) is a conformal primitive of $(K, k)$ in the following sense:

$$
\left.(d / d \varepsilon)\right|_{\varepsilon=0} \log \frac{\operatorname{det} \Delta[\omega+\epsilon \eta]}{\operatorname{det} \Delta[0]}=-\frac{1}{6 \pi}\left\{\int_{M} \eta(K d v)[\omega]+\oint_{\partial M} \eta(k d s)[\omega]\right\},
$$

where $\oint_{\partial M}(k d s)[\omega+\varepsilon \eta]$ is fixed. The total curvature, however, becomes a topological invariant by Gauss-Bonnet formula. Osgood, Phillips and Sarnak in [OPS-1] [OPS-2] [OPS-3] have proved the existence of extrema of the zeta functional determinant under conformal variations of both types (see also [PO] [On]). A crucial tool which they have used to establish their compactness result is the following sharp inequalites of Moser-Trudinger type

$$
\log \int_{S_{+}^{2}} e^{2 \phi} d v \leq \frac{1}{2 \pi} \int_{S_{+}^{2}}|\nabla \phi|^{2} d v+2 \int_{S_{+}^{2}} \phi \frac{d v}{2 \pi}
$$

and of Lebedev-Milin type

$$
\log \oint_{\partial D} e^{(u-\bar{u})} \frac{d \theta}{2 \pi} \leq \frac{1}{4}\left\{\int_{D} u \Delta u \frac{d x d y}{\pi}+2 \oint_{\partial D} u \frac{\partial}{\partial n} u \frac{d \theta}{2 \pi}\right\},
$$


where $\bar{u}$ is the average of $u$ on the boundary $\partial D$. Existence results in [OPS-1] also provide a new way to the understanding of the uniformization theorem for compact surfaces. Another remarkable result in [OPS-2] is that by observing that zeta functional determinant is a spectral information, they have derived from from inequalities (2.1.4) and (2.1.5) that isospectral set of metrics on compact surfaces is $\mathcal{C}^{\infty}$ compact.

In $[\mathrm{BCY}][\mathrm{CY}]$, results similiar to those of Osgood, Phillips and Sarnak have been established on compact 4-manifolds without boundary. Our goal here is to establish similiar results on compact manifolds with boundary.

For the purpose of stating our results more clearly, we shall modify the notions of two types of conformal variations which are more restrictive than the original definitions.

Definition 2.1.1. Suppose that $(M, g)$ is a 4-manifold with boundary $\partial M$ and that $H=0$. Then a metric $g_{\omega}=e^{2 \omega} g$ is called a conformal variation of type I if

(1) $\operatorname{vol}(M)[\omega]=\operatorname{vol}(M)[0]$

(2) $H[\omega]=H[0]=0$

(3) $\oint_{\partial M}(T d s)[\omega]=\oint_{\partial M}(T d s)[0]$.

Definition 2.1.2. Suppose that $(M, g)$ is a 4-manifold with boundary $\partial M$ and that $H=3$. Then a metric $g_{\omega}=e^{2 \omega} g$ is called a conformal variation of type II if

1. $\operatorname{vol}(\partial M)[\omega]=\operatorname{vol}(\partial M)[0]$

2. $H[\omega]=H[0]=3$

3. $\oint_{\partial M}(T d s)[\omega]=\oint_{\partial M}(T d s)[0]$.

\subsection{Conformal variations of type I}

Let us start with an example. Let $M=S_{+}^{4}$ with its standard metric. As a consequence of Theorem 1.3.4.in the first part of this article, we have in this special case the following Alvarez-Polyakov formula:

$$
\begin{aligned}
F[\omega]= & \beta_{2}\left\{-\pi^{2} \log \int_{S_{+}^{4}} e^{4(\omega-\bar{\omega})} d x+\frac{1}{4} \int_{S_{+}^{4}}\left(|\Delta \omega|^{2}+2|\nabla \omega|^{2}\right) d x\right\} \\
& +\beta_{3}\left\{\frac{1}{2} \int_{S_{+}^{4}}\left(J^{2} d x\right)[\omega]-\frac{1}{2} \int_{S_{+}^{4}}\left(J^{2} d x\right)[0]\right\}
\end{aligned}
$$

By considering the doubling space $S^{4}$ of $S_{+}^{4}$, where we have the sharp inequality of Beckner [Be-2] and the sharp Sobolev inequality of ObataSchoen $[\mathrm{Ob}][\mathrm{Sc}]$, we may conclude the following result. 
Theorem 2.2.1. On $S_{+}^{4}$ with standard metric as the background metric, within conformal variations of type $I, F[\omega] \leq 0$ when $\beta_{2}, \beta_{3}<0$, and the $F[\omega]=0$ is attained only when $e^{2 \omega} g_{0}=\phi^{*} g_{0}$ for some conformal transformation $\phi$ of the standard sphere $S^{4}$ which maps the upper hemisphere to itself.

It turns out that we can establish the existence of extremum of the functional determinant and $W^{2,2}$-compactness of isospectral set within conformal variations of type I on general 4-manifolds by applying the following sharp inequality of Adams type for Neumann problem.

Lemma 2.2.2. For any function $\phi$ with Neumann boundary condition $N \phi$ $=0$, on a given 4-manifolds $M$ with smooth boundary $\partial M$, one has

$$
\int_{M} e^{\alpha|\phi-\bar{\phi}|^{2}} d v \leq C(M, \alpha) \text { if } \int_{M}|\Delta \phi|^{2} d v \leq 1
$$

for any $\alpha<16 \pi^{2}$. Consequently one has

$$
\int_{M} e^{4(\phi-\bar{\phi})} d v \leq \frac{4}{\alpha} \int_{M}|\Delta \phi|^{2} d v+\log C(M, \alpha)
$$

(2.2.1) is an analogue of Moser-Adams inequality [M] [T] [Ad]. The idea of the proof of the inequality is first to embed $M$ into a bigger manifold $\tilde{M}$ as an open domain, then to construct the Green function (cf. [Au]) with respect to Neumann condition with the assistance of reflections defined in small tubular neighborhoods of $\partial M$ in $\tilde{M}$, finally using reflections to convert this problem into that where one may apply Moser-Adams inequality [Ad]. We would like to remark that although our proof does establish $16 \pi^{2}$ as the best exponent in the equality (2.2.1), we do not know if this exponent can be attained as in the case of Adam's original inequality for the corresponding Dirichlet case.

In the following discussions, we shall name

$$
a_{4}(A, B)=\beta_{1} \int|C|^{2}+\beta_{2}\left\{\frac{1}{2} \int Q+\oint T\right\}+\beta_{4} \oint \mathcal{L}_{4}+\beta_{5} \oint \mathcal{L}_{5}
$$

the conformal index of the operator $(A, B)$. Notice that $a_{4}(A, B)$ does not depend on the choice of the background metric for metrics in the same conformal class. As we shall see in the following results, $a_{4}(A, B)$ plays the same role as the corresponding index $a_{4}(A)$ (which was denoted by $k_{d}(A)$ in $[\mathrm{CY}]$ ) does for compactness results on zeta functional determinants. As a consequence of the sharp inequality $(2.2 .2)$ we have the following compactness result. 
Theorem 2.2.3. Suppose that $(A, B)$ satisfies the three assumptions. And suppose that $\beta_{2}, \beta_{3}<0$, and $\frac{1}{\beta_{2}} a_{4}(A, B)<4 \pi^{2}$. Then within conformal variations of type $I, F[\omega]$ is bounded from above and

$$
\left\|\omega-\omega_{b}\right\|_{W^{2,2}}^{2}+\left\|\omega-\omega_{b}\right\|_{W^{1,4}}^{4} \leq C|F[\omega]|+C
$$

where $\omega_{b}$ is the average of $\omega$ over $\partial M$ and $C$ does not depend on $\omega$.

Since we can control $\omega_{b}$ through the spectral information of the metric $g_{w}$, we have as a direct consequence of Theorem (2.2.3) the following result.

Corollary 2.2.4. Suppose that $(A, B)$ satisfies the three assumptions. And suppose that $-a_{4}(A, B)<\left(-\beta_{2}\right) 4 \pi^{2}$ and $\beta_{2}, \beta_{3}<0$. Then within conformal variations of type $I$, the metrics $g_{\omega}$ with the same spectrum as $g_{0}$ satisfy the estimate

$$
\|\omega\|_{W^{2,2}} \leq C
$$

where $C$ is a constant which does not depend on $\omega$.

Before discussing the extremum property of log determinant $F[\omega]$, we would like to remark that although we have $W^{2,2}$ bound for metrics $g_{w}$ conformal variation Type I, in general this class of metrics is not closed under the weak limit of $W^{2,2}$ norms. For this reason, we need to further restrict our class of metrics.

Definition 2.2.5. We call $g_{\omega}$ a restricted conformal variation of type $I$ from $g_{0}$ if it is a conformal variation of type $I$ and $P_{4}[0](\omega)=0$ in $M$.

We would like to remark that the additional condition that $P_{4}[0](\omega)=0$ is similar to the condition that the metric $g_{w}$ being flat imposed in the works of [OPS-2], [OPS-3] on compact surfaces.

Corollary 2.2.6. Suppose that $(A, B)$ satisfies the three assumptions. And suppose that $-a_{4}(A, B)<\left(-\beta_{2}\right) 4 \pi^{2}$ and $\beta_{2}, \beta_{3}<0$. Within restricted conformal variations of type $I, F[\omega]$ has its maximum achieved by some $\omega_{d} \in W^{2,2}$.

We also established the uniqueness of the extremal metric of functional determinant if it exists, as a consequence of the convexity property of functional determinant(cf. $[\mathrm{CY}]$ ).

Theorem 2.2.7. Suppose that $(A, B)$ satisfies the three assumptions, and suppose that $2 \beta_{3} \leq \beta_{2} \leq \beta_{3}<0$, and $a_{4}(A, B) \geq 0$. In addition assume 
that $\left(\beta_{2}+6 \mu_{4}\right) L[0]$ is nonnegative definite. Then the maximum of $F[\omega]$ under conformal variations of type $I$ is unique when it exists.

Example 2.2.8. We have some simple examples where all assumptions in Theorem 2.2.7 above are satisfied. We will consider the boundary value problems where $A=\mathcal{L}$ and $B$ is either Dirichlet or Robin boundary operator. In this case $\beta_{1}=2$ and $\beta_{2}=\beta_{3}=-8$ (see Table 1.3.8). Apply Chern-Gauss-Bonnet Formula, we obtain:

(1) For $S^{2} \times S_{+}^{2}$, we have vol $=8 \pi^{2}, \chi=2,|C|^{2}=\frac{16}{3}$, and $L=0$. Therefore we have $a_{4}=\frac{128}{3} \pi^{2}>0$

(2) For $\Sigma_{g} \times S_{+}^{2}$, where $\Sigma_{g}$ is a hyperbolic surface of genus $g>1$, we know $\chi=2-2 g,|C|=0$, and $L=0$. Therefore $a_{4}=64(g-1) \pi^{2}>0$.

\subsection{Conformal variation of type II}

The problems involved in the study of conformal variations of type II is in general more difficult than that of the study of conformal variations of type I. We will demonstrate, in the special case when $M=B^{4}$, some geometric and analytic aspects to study the zeta functional determinant under conformal variation of this type. We hope that the discussion of this special case will shed light for the study of the problem for general manifolds. We now fix the standard metric of $B^{4}$ as the background metric $g_{0}$ and write the Alvarez-Polyakov formula on $B^{4}$.

Lemma 2.3.1. Suppose that $(A, B)$ satisfy the three assumptions. Let $(M, \partial M)$ be $\left(B^{4}, S^{3}\right)$ with the standard metric $g_{0}$. If $g_{\omega}$ is a conformal variation of type II, then the Alvarez-Polyakov formula is

$$
\begin{aligned}
F[\omega]= & -\frac{1}{2 l} \log \frac{\operatorname{det}(A, B)[\omega]}{\operatorname{det}(A, B)[0]} \\
= & \beta_{2} D[\omega]+\frac{1}{2} \beta_{3} \int_{B^{4}} J^{2}[\omega] e^{4 \omega}+3 \mu_{3} \oint_{S^{3}}(\tau-3 F)[\omega] e^{3 \omega} d y \\
& +3 \mu_{2}\left\{\oint_{S^{3}} \tilde{\tau}[\omega] e^{3 \omega} d y-\oint_{S^{3}} 6 d y\right\}
\end{aligned}
$$

where

$$
\begin{aligned}
D[\omega]= & \frac{1}{4} \int_{B^{4}} \omega\left(P_{4} \omega\right) d x+\frac{1}{2} \oint_{S^{3}} \omega\left(P_{3} \omega\right) d y \\
& +2 \oint_{S^{3}} \omega d y-\frac{1}{4} \oint_{S^{3}}\left(\omega_{N}+\omega_{; N N}\right) d y
\end{aligned}
$$

and

$$
P_{3}=-\frac{1}{2} N \Delta-\tilde{\Delta} N+\tilde{\Delta} \text { and } P_{4}=\Delta^{2}
$$


In the discussions below, the following sharp inequality of LebedevMilin type plays the same role for the compactness results as the classical Lebedev-Milin inequality does for compact surface with boundary.

Lemma 2.3.2. Suppose $\omega \in C^{\infty}\left(\overline{B^{4}}\right)$, and assume $\omega$ satisfies

(1) $H[\omega]=H[0]=3$ (i.e., $\left.N \omega\right|_{S^{3}}=1-e^{\omega}$ ),

$(2) \oint_{S^{3}}(\tau d y)[\omega]=\oint_{S^{3}}(\tau d y)[0]=0$

Then

$$
\log \left\{\frac{1}{2 \pi^{2}} \oint_{S^{3}} e^{3 \omega} d y\right\} \leq \frac{3}{4 \pi^{2}} D[\omega]
$$

or equivalently

$$
\begin{array}{r}
\log \left\{\frac{1}{2 \pi^{2}} \oint_{S^{3}} e^{3(\omega-\bar{\omega})} d y\right\} \leq \frac{3}{4 \pi^{2}}\left\{\frac{1}{4} \int_{B^{4}} \omega P_{4} \omega d x+\frac{1}{2} \oint_{S^{3}} \omega P_{3} \omega d y\right. \\
\left.-\frac{1}{4} \oint_{S^{3}}\left(\omega_{N}+\omega_{; N N}\right) d y\right\}
\end{array}
$$

where $\bar{\omega}=\frac{1}{2 \pi^{2}} \oint_{S^{3}} \omega d y$. Moreover the equality holds if and only if $e^{2 \omega} g_{0}$ is isometric to $g_{0}$.

The proof of this sharp inequality consists of two steps. The first step is to prove

$$
\begin{array}{r}
\frac{1}{2} \oint_{S^{3}} \omega \mathcal{P}_{3} \omega d y \leq \frac{1}{4} \int_{B^{4}} \omega P_{4} \omega d x+\frac{1}{2} \oint_{S^{3}} \omega P_{3} \omega d y \\
-\frac{1}{4} \oint_{S^{3}}\left(\omega_{; N N}+\omega_{N}\right) d y
\end{array}
$$

where

$$
\mathcal{P}_{3} u=\tilde{\Delta}(\tilde{\Delta}+1)^{\frac{1}{2}} u,
$$

is the so called Paneitz operator on $S^{3}$ (cf. [Be-2] [B-1] [B-2]). The second step is to apply the following sharp inequality of Beckner [Be-2]

$$
\log \left\{\frac{1}{2 \pi^{2}} \oint_{S^{3}} e^{3(\omega-\bar{\omega})} d y\right\} \leq \frac{3}{8 \pi^{2}} \oint_{S^{3}} \omega \mathcal{P}_{3} \omega d y
$$

To establish the inequality (2.3.4), we apply the following analytic result.

Lemma 2.3.3. Suppose $w$ satisfies

$$
\begin{aligned}
\Delta^{2} w & =0, \text { on } B^{4} \\
\left.w\right|_{\partial B^{4}} & =u \\
\left.N w\right|_{\partial B^{4}} & =\phi .
\end{aligned}
$$


Then we have

$$
P_{3} \omega=\mathcal{P}_{3} \omega \text { on } S^{3}
$$

and

$$
\omega_{; N N}+\tilde{\Delta} \omega=-2 \mathcal{P}_{1} N \omega-N \omega, \text { on } S^{3},
$$

where $\mathcal{P}_{1}=\left\{(\tilde{\Delta}+1)^{\frac{1}{2}}-1\right\}$ is the so-called Dirichlet-Neumann operator on $B^{4}$.

As a consequence of (2.3.3') and the sharp Sobolev inequality on $S^{3}$, we can establish the following theorem.

Theorem 2.3.4. Suppose that $(A, B)$ satisfies the three assumptions, and that $(A, B)$ has no zero eigenvalue. Let $(M, \partial M)$ be $\left(B^{4}, S^{3}\right), g_{\omega}$ be a conformal variation of type II defined on $B^{4}$. Assume further that $g_{\omega}$ satisfies $\oint_{S^{3}}\{(\tau d y)[\omega]-(\tau d y)[0]\}=0$, and that $\beta_{2}, \beta_{3}$ and $\mu_{2}+\frac{3}{2} \mu_{3}$ have the same sign. Then the zeta functional determinant maintains the same sign and attains zero at and only at the metrics isometric to the standard metric.

Example 2.3.5. Recall from Table 1.3.8 in the first part of this article that $\beta_{2}=-8, \beta_{3}=-8$, and $3 \mu_{2}+\frac{9}{2} \mu_{3}=-29$ when $A=\mathcal{L}$ the conformal laplacian and $B=\mathcal{R}$ the Robin boundary operator. Therefore $(\mathcal{L}, \mathcal{R})$ satisfies all the assumptions in Theorem 2.3.4.

We now discuss the zeta functional determinants within another natural subclass of conformal variations of type II.

Definition 2.3.6. We call $g_{\omega}$ a restricted conformal variation of type $I I$ from $g_{0}$ if it is a conformal variation of type II and $P_{4}[0](\omega)=0$ in $M$.

For example, when $\left(M, g_{0}\right)=\left(B^{4}, g_{0}\right), P_{4}[0](\omega)=(\Delta)^{2}[0](\omega)$. Metrics $g_{\omega}$ conformal variation of type II are in the restricted class if and only if $w$ is biharmonic on $B^{4}$.

Theorem 2.3.7. Suppose that $(A, B)$ satisfies the three assumptions. Assume further that $(A, B)$ has no zero eigenvalue. Let $(M, \partial M)$ be $\left(B^{4}, S^{3}\right)$. Then within the set of restricted conformal variations of type II, the zeta functional determinant stays in the same sign and attains zero at and only at metrics which are isometric to the standard metric, provided that $\beta_{2}, \beta_{3}, \mu_{2}, \mu_{3}$ have the same sign.

This result is established as a consequence of three sharp inequalities: the sharp inequality of Beckner (2.3.5) [Be-2], the sharp Sobolev inequality of Obata-Schoen on $S^{3}$, and the sharp Sobolev trace inequality due to Escobar and Beckner (2.1.2) [E-1] [Be-1]. It is very interesting to see that all these three sharp inequalities on $S^{3}$ appear simutaneously as parts of the log-determinant formula for $\left(B^{4}, S^{3}\right)$. 
Example 2.3.8. $\beta_{2}, \beta_{3}, \mu_{2}, \mu_{3}$ all are negative when $(A, B)=(\mathcal{L}, \mathcal{R})$. See Table 1.3.8 in the first part of this article. Meanwhile they are not of the same sign when $(A, B)=(\mathcal{L}, \mathcal{D})$.

\section{References}

[Ad] D. Adams, A sharp inequality of J. Moser for higher order derivatives, Ann. of Math. 128 (1988), 385-398.

[Al] O. Alvarez, Theory of strings with boundary, Nucl. Phys. B 216 (1983), 125184.

[Au] T. Aubin, Nonlinear Analysis on Manifolds, Monge-Ampere equations, Springer Verlag, 1982.

[B-1] T. Branson, Group representations arsing from Lorentz conformal geometry, J. Funct. Anal. 74 (1987), 199-293.

[B-2] Sharp inequality, the functional determinant and the complementary series, preprint (1992).

[B-3] Personal communcation (1990).

[Be-1] W. Beckner, Conformal geometry and Escobar's Sobolev trace inequality, preprint.

[Be-2] Sharp Sobolev inequalities on the sphere and the Moser-Trudinger inequality, Ann. of Math. 138 (1993), 213-242.

[BCY] T. Branson, S-Y. A. Chang, and P. C. Yang, Estimates and extremal problems for the log-determinant on 4-manifolds, Comm. Math. Physics 149; No. 2 (1992), 241-262.

[BG-1] T. Branson and P. Gilkey, The functional determinant of a 4-dimensional boundary value problem, preprint (1992); to appear in Trans. Amer. Math. Soc..

[BG-2] The asymptotics of the Laplacian on a manifold with boundary, Comm. PDE 15 (1990), 245-272.

[BG-3] - The functional determinant in the standard conformal class on four dimensional balls and spherical shells, Proceedings of the $24^{\text {th }}$ National Conference on Geometry and Topology (Romania) (1994).

[BO ] T. Branson and B. Ørsted, Explicit functional determinants in four dimensions, Proc. Amer. Math. Soc. 113 (1991), 901-920.

[CQ-1] S-Y. A. Chang and J. Qing, The zeta functional determinants on 4-manifolds with boundary I - the formula, preprint.

[CQ-2] , The zeta functional determinants on 4-manifolds with boundary II extremal metrics and and compactness of isospectral set, preprint (1995).

[CY] S-Y. A. Chang and P. C. Yang, Extremal metrics of zeta functional determinants on 4-manifolds, Ann. of Math. 145 (1995), 171-212.

[E-1] J. Escobar, Sharp constant in a Sobolev trace inequality, J. Diff. Geom. 37 (1992), 687-698.

[E-2] The Yamabe problem on manifolds with boundary inequality, J. Diff. Geom. 35 (1992), 21-84.

[E-3] Conformal deformation of a Riemannian metric to a scalar flat metric with constant mean curvature on the boundary, Ann. of Math. 136 (1992), 150.

[F] L. Fontana, Sharp borderline Sobolev inequalities on compact Riemannian Manifolds, Comm. Math. Helv. 68 (1993), 415-454. 
[M] J. Moser, A sharp form of an inequality by N. Trudinger, Indian Math. J. 20 (1971), 1077-1091.

[Ob] M. Obata, Certain conditions for a Riemannian manifold to be isometric with a sphere, J. Math. Soc. Japan 14 (1962), 333-340.

[On] E. Onofri, On the positivity of the effective action in a theory of random surfaces, Comm. Math. Phys. 86 (1982), 321-326.

[OPS-1] B. Osgood, R. Phillips, and P. Sarnak, Extremals of determinants of Laplacians, J. Funct. Anal. 80 (1988), 148-211.

[OPS-2] _ Compact isospectral sets of surfaces, J. Funct. Anal. 80 (1988), 212234.

[OPS-3] _ Moduli space, heights and isospectral sets of plane domians, Ann. of Math. 129 (1989), 293-362.

[Po] A. Polyakov, Quantum geometry of Bosonic strings, Phys. Lett. B 103 (1981), 207-210.

[Sc] R. Schoen, Conformal deformation of a Riemannian metric to constant scalar curvature, J. Diff. Geom. 20 (1984), 479-495.

[T] N. Trudinger, Remarks concerning the conformal deformation of Riemannian structure on compact manifolds, Ann. Scuo. Norm, Sup. Pisa 3 (1968), 265-274.

Sun-Yung A. Chang, Department of Mathematics, Univerity of CaliforNIA, LOS ANGELES, CA 90095

E-mail address: chang@math.ucla.edu

Jie Qing, Department of Mathematics, Columbia Univerity, New York, N Y 10027

E-mail address: qing@math.columbia.edu 